Original Article

\title{
Rapamycin reduces orofacial nociceptive responses and microglial p38 mitogen-activated protein kinase phosphorylation in trigeminal nucleus caudalis in mouse orofacial formalin model
}

\author{
Ji-Hee Yeo, Sol-Ji Kim, and Dae-Hyun Roh* \\ Department of Oral Physiology, School of Dentistry, Kyung Hee University, Seoul 02447, Korea
}

\section{ARTICLE INFO}

Received February 25, 2021

Revised May 7, 2021

Accepted May 21, 2021

\section{*Correspondence}

Dae-Hyun Roh

E-mail:dhroh@khu.ac.kr

Key Words

Glia

mTOR

Orofacial pain

p38 mitogen-activated protein kinases Rapamycin

\begin{abstract}
The mammalian target of rapamycin (mTOR) plays a role in various cellular phenomena, including autophagy, cell proliferation, and differentiation. Although recent studies have reported its involvement in nociceptive responses in several pain models, whether mTOR is involved in orofacial pain processing is currently unexplored. This study determined whether rapamycin, an mTOR inhibitor, reduces nociceptive responses and the number of Fos-immunoreactive (Fos-ir) cells in the trigeminal nucleus caudalis (TNC) in a mouse orofacial formalin model. We also examined whether the glial cell expression and phosphorylated p38 (p-p38) mitogen-activated protein kinases (MAPKs) in the TNC are affected by rapamycin. Mice were intraperitoneally given rapamycin $(0.1,0.3$, or $1.0 \mathrm{mg} / \mathrm{kg})$; then, $30 \mathrm{~min}$ after, $5 \%$ formalin (10 $\mu$ ) was subcutaneously injected into the right upper lip. The rubbing responses with the ipsilateral forepaw or hindpaw were counted for $45 \mathrm{~min}$. High-dose rapamycin $(1.0 \mathrm{mg} / \mathrm{kg})$ produced significant antinociceptive effects in both the first and second phases of formalin test. The number of Fos-ir cells in the ipsilateral TNC was also reduced by high-dose rapamycin compared with vehicle-treated animals. Furthermore, the number of p-p38-ir cells the in ipsilateral TNC was significantly decreased in animals treated with high-dose rapamycin; $p$-p38 expression was co-localized in microglia, but not neurons and astrocytes. Therefore, the mTOR inhibitor, rapamycin, reduces orofacial nociception and Fos expression in the TNC, and its antinociceptive action on orofacial pain may be associated with the inhibition of p-p38 MAPK in the microglia.
\end{abstract}

\section{INTRODUCTION}

The orofacial area is one of the most densely innervated areas by the trigeminal nerve and thus becomes a focus for some of the most common types of acute pain, such as in pathological conditions of the tooth and its associated structures [1]. However, only limited studies have assessed pain relief in the trigeminal region. Thus, there are many difficulties in the management of both acute and chronic orofacial pain conditions, owing to insufficient awareness and understanding of its pain mechanisms [2,3]. Pain management in orofacial disorders is one of the most difficult areas of study in pharmacology.

Mammalian target of rapamycin (mTOR) is one of the fundamentally important complexes in cell biology and cellular signaling mechanisms. It is involved in cell growth regulation, proliferation and migration, lipid synthesis, and ribosomal bioproduction and autophagy. It is expressed in all cells as a protein synthesis regulator, which is essential for normal cell development [3-5]. mTOR inhibition leads to anti-inflammatory, antifibrotic, antitumor, and antifungal properties; mTOR signaling

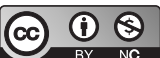

This is an Open Access article distributed under the terms of the Creative Commons Attribution Non-Commercial License, which permits unrestricted non-commercial use, distribution, and reproduction in any medium, provided the original work is properly cited. Copyright @ Korean J Physiol Pharmacol, pISSN 1226-4512, elSSN 2093-3827
Author contributions: J.H.Y. and S.J.K. performed all experiments. J.H.Y. and D.H.R. designed the study, analyzed the data, and drafted the manuscript. D.H.R. completed the manuscript. 
involvement has been established in a variety of cellular functions [6,7]. Recently, it has been reported that mTOR inhibition produces antipruritic, anti-inflammatory, and analgesic effects in various rodent models for inflammatory, itching, and neuropathic pain [8-10]. However, whether mTOR signaling contributes to the induction of orofacial pain and whether mTOR inhibition can exert analgesic effects for orofacial pain remain unclear.

The activation of glial cells and neuroglial interactions are emerging as key mechanisms underlying chronic pain [11]. The current evidence is primarily associated with two types of glial cells in the development of inflammatory or neuropathic pain: microglia and astrocytes of the central nervous system (CNS), such as the spinal cord and trigeminal nucleus caudalis (TNC) [11-13]. In addition, mitogen-activated protein kinases (MAPKs) play a crucial role in cell signaling and gene expression in the CNS. MAPKs are activated by phosphorylation and function as regulators of transcription and non-transcription, converting a wide range of extracellular stimuli into a variety of intracellular stimuli [14]. In particular, the phosphorylated p38 (p-p38) MAPK in spinal dorsal horn cells plays an important role in the induction and maintenance of pain hypersensitivity [14,15].

This study aimed to verify whether rapamycin, a well-known mTOR inhibitor, could reduce nociceptive responses and the number of Fos-immunoreactive (Fos-ir) cells in the TNC in a mouse orofacial formalin model. We also examined whether the activation of glial cells (astrocytes and microglia) and p-p38 MAPK in TNC were modified by rapamycin treatment.

\section{METHODS}

\section{Animals}

Experiments were performed using male C57BL/6 mice (25-30 $\mathrm{g}$; DBL, Seoul, Korea) housed in colony cages with free access to food and water and maintained in temperature- and light-controlled rooms $\left(23^{\circ} \mathrm{C} \pm 2^{\circ} \mathrm{C}, 12 / 12\right.$-h light/dark cycle with lights on at 07:00) for at least 1 week prior to the experiment. The experimental protocols for animal usage were reviewed and approved by the Kyung Hee University Institutional Animal Care and Use Committee (KHUASP(SE)-16-014) and conformed to the guidelines of the National Institutes of Health (NIH publication no. 8623, revised 1985).

\section{Drugs}

Rapamycin (LC laboratories, Woburn, MA, USA), an mTOR inhibitor, was prepared in dimethyl sulfoxide (DMSO) as a stock solution at $100 \mathrm{mg} / \mathrm{ml}$ and was then dissolved in physiological saline at concentrations of $0.1,0.3$, and $1.0 \mathrm{mg} / \mathrm{kg}$ immediately before injection. These doses of rapamycin were selected based on previous studies $[16,17]$. The final vehicle (VEH) was adjusted to $0.2 \%$ DMSO in saline. Mice were weighted and then injected intraperitoneally (i.p.) $30 \mathrm{~min}$ prior to formalin injection.

\section{Formalin-induced orofacial pain test}

The orofacial formalin test was performed as previously described $[18,19]$. Formalin was prepared from commercially available stock formalin (aqueous solution of $37 \%$ formaldehyde; JUNSEI, Tokyo, Japan) and further diluted to $5 \%$ in physiological saline. Mice were first acclimatized for $30 \mathrm{~min}$ in an acrylic observation chamber $(15 \times 15 \times 15 \mathrm{~cm})$. Afterward, the mice received $10 \mu \mathrm{l}$ subcutaneous (s.c.) injection of $5 \%$ formalin through a 30 -gauge needle attached to Hamilton syringe into the right upper lip, lateral to the nose. Next, the animals were immediately placed back into the acrylic chamber, and nociceptive responses in each animal were recorded using a video camera for a 45-min observation period. The recording was divided into 15 blocks of $3 \mathrm{~min}$, and a nociceptive score was determined for each block by measuring the number of seconds the animal spent grooming the injected area with the ipsilateral forepaw or hindpaw. The movements of the ipsilateral forepaw were accompanied by the movements of the contralateral forepaw. The duration of the responses during the first 2 blocks represented the first phase (0-6 min postinjection), whereas the duration of responses during the subsequent 13 blocks represented the second phase (6-45 min postinjection) in the formalin-induced orofacial pain test $[1,19]$. The behavior was analyzed by an investigator who was blinded to the animal's group assignment.

\section{Immunohistochemistry}

TNC immunohistochemistry was performed as previously described $[19,20]$. Fos, a common marker of neuronal activation, is gradually activated by physiological stimuli such as stress and inflammation. Fos mRNA accumulates and peaks 30-45 min after stimulation, whereas Fos protein synthesis takes about 1-2 $\mathrm{h}$ after stimulation before reaching its peak value [21,22], In addition, the p-p38 MAPK increases within minutes, persisting for hours or days after tissue injury [22-24]. Thus, the animals were sacrificed $2 \mathrm{~h}$ after formalin injection, and immunohistochemistry was performed. Animals were deeply anesthetized with $5 \%$ isoflurane and transcardially perfused through the ascending aorta with $0.1 \mathrm{M}$ phosphate-buffered saline (PBS, $50 \mathrm{ml}, \mathrm{pH}$ 7.4), followed by $4 \%$ paraformaldehyde. After perfusion, the brainstem was immediately removed and stored overnight at $4^{\circ} \mathrm{C}$ in the same fixative and then placed in a cryoprotectant solution $(30 \%$ sucrose in PBS) for at least two nights at $4^{\circ} \mathrm{C}$ before sectioning. Serial transverse sections $(30 \mu \mathrm{m})$ were cut from the TNC using a cryostat (Leica Microsystems, Wetzlar, Germany) and collected in PBS. After eliminating endogenous peroxidase activity with 3\% hydrogen peroxide in PBS and pre-blocking with 3\% normal goat serum and $0.3 \%$ Triton X-100 in PBS, sections were incubated in 
polyclonal rabbit anti-Fos antibody (1:1,000; Santa Cruz Biotechnology Inc., Santa Cruz, CA, USA) overnight at $4^{\circ} \mathrm{C}$. After washing several times with PBS, the tissue sections were incubated with biotinylated anti-rabbit antibody (1:200; Vector Laboratories, Burlingame, CA, USA) for $1 \mathrm{~h}$ at room temperature; then, the tissue sections were processed using the avidin-biotin method (Elite ABC; Vector Laboratories). Finally, Fos-ir cells were visualized using a 3-3 diaminobenzidine reaction intensified with $0.2 \%$ nickel chloride.

For staining experiments involving glial fibrillary acidic protein (GFAP), ionized calcium-binding adapter molecule-1 (Iba-1), neuronal nuclear protein (NeuN), and p-p38, after preblocking with $5 \%$ normal donkey serum plus $0.3 \%$ triton X-100 in PBS at room temperature for $1 \mathrm{~h}$, free-floating TNC sections were incubated overnight at $4^{\circ} \mathrm{C}$ with primary antibodies against GFAP (1:1,000; Cell Signaling, Danvers, MA, USA), Iba-1 (1:500; Wako, Tokyo, Japan), and NeuN (1:1,000; Millipore, Billerica, MA, USA), respectively. These were also co-incubated with rabbit antibody against p-p38 (1:1,000; Cell Signaling) in 1\% normal donkey serum plus $0.3 \%$ Triton X-100 in PBS at $4^{\circ} \mathrm{C}$ overnight. After washing several times with PBS, the tissue sections were incubated with Cy3-conjugated secondary anti-rabbit antibody (1:500; Jackson ImmunoResearch, West Grove, PA, USA) and Alexa Fluor 488-conjugated secondary anti-mouse or anti-goat antibodies (1:500 or 1:400; Jackson ImmunoResearch) for $4 \mathrm{~h}$ at room temperature. After washing several times with PBS, the tissue sections were mounted onto slides under a cover slip.

\section{Image analysis}

The TNC tissues were scanned using the ECLIPSE 80i (Nikon Corp., Kanagawa, Japan) brightfield and fluorescent microscope, and individual sections were digitized with 4096 gray levels using a cooled CCD camera (Cool Snap ES model; Nihon Roper, Tokyo, Japan) connected to a computer-assisted image analysis system (MetaMorph; Universal Imaging, Westchester, PA, USA). For quantitative analysis in the TNC region, six nonadjacent tissue sections per each mouse were randomly selected and analyzed using a computer-assisted image analysis system (MetaMorph version 7.7.2.0; Universal Imaging). For Fos image analysis, we set the shape factor to $0.5-1.0$ and counted only the cells that were at least 30\% darker than the average level of each image [20]. For p-p38, we counted cells that were at least $80 \%$ brighter than the average level of each image. To analyze GFAP- and Iba-1-ir images, we measured the positive pixel areas that had an $80 \%$ brightness level within the range of intensity levels [25]. The average of percent threshold area of immunoreactivity per section from each animal was obtained, and these values were averaged across each group and presented as group data. All analytical procedures described above were blindly performed without knowledge of the experimental conditions.

\section{Statistical analysis}

All values are expressed as mean \pm SEM. Data analysis and statistical comparisons were performed using GraphPad Prism version 6.0 (GraphPad Software, San Diego, CA, USA). For multiple comparisons in the formalin pain behavior test, either two-way repeated measures ANOVA or one-way ANOVA followed by post hoc Bonferroni test was performed. The data from immunohistochemistry was analyzed using one-way ANOVA, followed by post hoc Bonferroni test. Statistical significance was set at $\mathrm{p}<0.05$.

\section{RESULTS}

\section{Rapamycin reduces orofacial formalin-induced nociceptive responses}

Rapamycin was i.p. injected at concentrations of $0.1,0.3$, and 1.0 $\mathrm{mg} / \mathrm{kg} 30 \mathrm{~min}$ prior to s.c. facial injection of $5 \%$ formalin. Mice that were i.p. injected in the VEH group exhibited typical biphasic pain behaviors during the 45 -min observation period (first phase: 0-6 min after formalin injection; second phase: 6-45 min after formalin injection). The injection of low and medium dose of rapamycin ( 0.1 and $0.3 \mathrm{mg} / \mathrm{kg}$, respectively) did not suppress orofacial formalin-induced nociceptive responses compared with the VEH group. On the other hand, the nociceptive responses in the high-dose rapamycin group $(1.0 \mathrm{mg} / \mathrm{kg})$ significantly decreased at 3, 18-30, and $42 \mathrm{~min}$ after formalin injection (Fig. 1A). Furthermore, high-dose rapamycin $(1.0 \mathrm{mg} / \mathrm{kg})$ significantly reduced formalin-induced nociception in the first and second phases of the formalin test (Fig. 1B).

\section{Rapamycin inhibits the increase of Fos-ir cells in TNC}

We examined the number of Fos-ir cells in ipsilateral TNC region $2 \mathrm{~h}$ after orofacial formalin injection. In Fig. 2, the number of Fos-ir cells was increased in the TNC of the VEH group before orofacial formalin injection. Although low- and medium-dose rapamycin $(0.1$ and $0.3 \mathrm{mg} / \mathrm{kg}$, respectively) did not reduce the number of Fos-ir cells compared with the VEH group (Fig. 2A$\mathrm{C}, \mathrm{E})$, high-dose rapamycin $(1.0 \mathrm{mg} / \mathrm{kg})$ significantly suppressed orofacial formalin-induced Fos expression in the TNC (Fig. 2D, E).

\section{Rapamycin does not modify GFAP and Iba-1 expression in TNC}

The change of expression of astrocytes (GFAP) and microglia (Iba-1) in the TNC region after rapamycin treatment was examined in orofacial formalin-injected mice. In Fig. 3, it was confirmed that both the astrocyte marker, GFAP (Fig. 3A-E), and microglia marker, Iba-1 (Fig. 3F-J), did not change in the VEH group or in any of the rapamycin-treated groups $(0.1,0.3$, and $1.0 \mathrm{mg} / \mathrm{kg})$. 


\section{A}

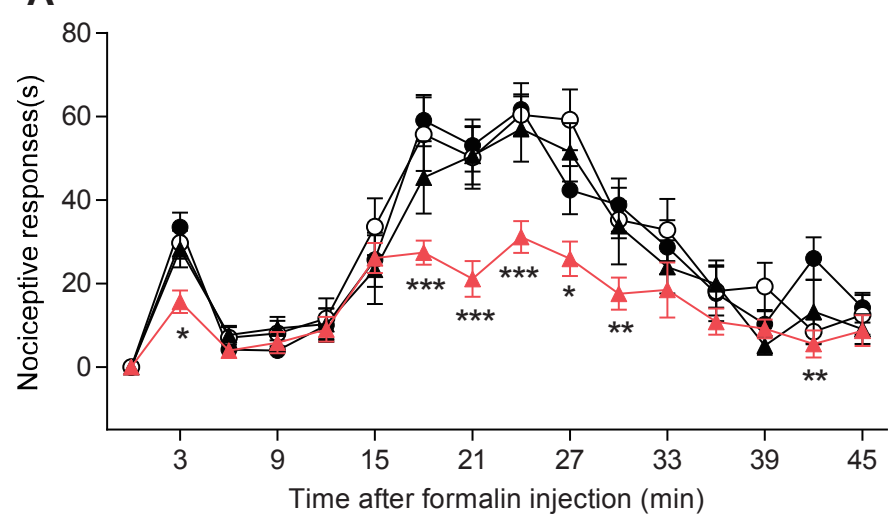

VEH $(n=12)$

-O- $0.1 \quad(n=10)$

$\leftarrow 0.3 \quad(\mathrm{n}=10)$

$\leftarrow 1.0 \quad(\mathrm{n}=11)$

(mg/kg, i.p.)

B

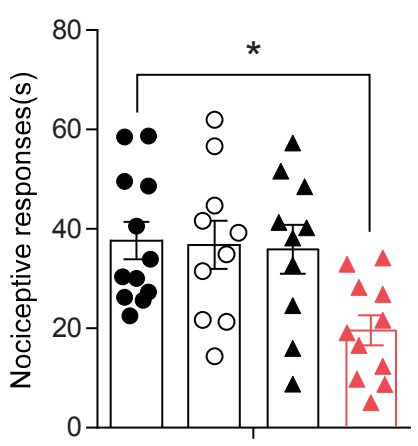

First phase
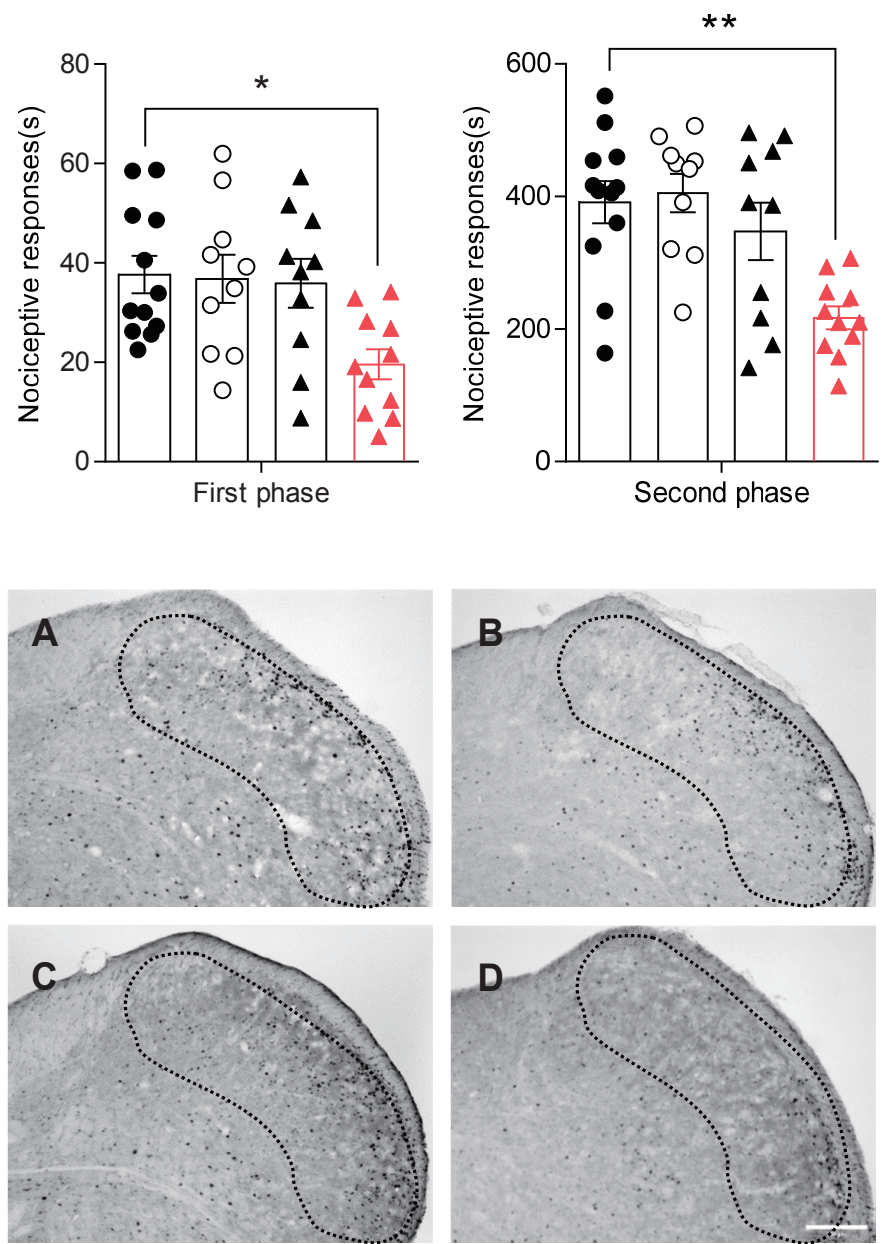

Second phase

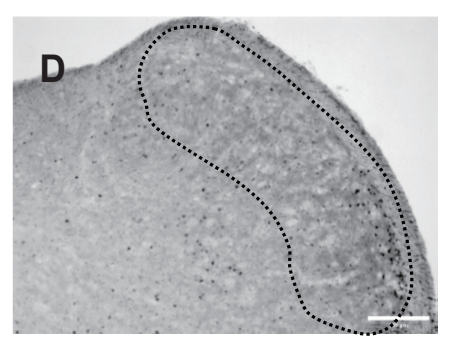

- $\operatorname{VEH}(n=12)$

O $0.1 \quad(n=10)$

\ $0.3 \quad(n=10)$

$\triangle 1.0 \quad(n=11)$ (mg/kg, i.p.)
Fig. 1. Effect of rapamycin in the orofacial formalin test in mice. Low- and medium-dose rapamycin ( 0.1 and 0.3 $\mathrm{mg} / \mathrm{kg}$, respectively) did not suppress orofacial formalin-induced nociceptive responses. On the other hand, highdose rapamycin $(1.0 \mathrm{mg} / \mathrm{kg})$ significantly reduced nociceptive response at 3 , 18-30, and $42 \mathrm{~min}$ after formalin injection (A). High-dose rapamycin (1.0 mg/ $\mathrm{kg}$ ) showed potent antinociceptive effects in the first and second phases (B) compared with the vehicle-treated (VEH) group $\left({ }^{*} p<0.005,{ }^{* *} p<0.01\right.$, and ${ }^{* * *} p$ $<0.001$, compared with the VEH group, $\mathrm{n}=10-12$ per group). i.p., intraperitoneally.

Fig. 2. Effect of rapamycin on the increase of Fos-immunoreactive (Fos-ir) cells in the trigeminal nucleus caudalis (TNC). The number of Fos-ir cells increased in the TNC of the vehicle-treated (VEH) group (A, E); low- and medium-dose rapamycin ( $0.1 \mathrm{and} 0.3 \mathrm{mg} / \mathrm{kg}$ ) did not suppress this increase of Fos-ir cells $(B, C, E)$. On the other hand, high-dose rapamycin $(1.0 \mathrm{mg} / \mathrm{kg})$ significantly reduced the increase of Fos-ir cells in the ipsilateral TNC (D, E, *** $p<0.001$, compared with the VEH group, $n=5$ ). The boundaries of the TNC are outlined by the dotted line. Scale bar $=200 \mu \mathrm{m}$.

\section{Rapamycin reduces the number of $p-p 38$ MAPK in TNC}

We also attempted to confirm whether the treatment of rapamycin could modify the p-p38 MAPK in orofacial formalin- injected mice. The number of p-p38 MAPK-ir cells in the ipsilateral TNC region did not change after low- and medium-dose rapamycin treatment $(0.1$ and $0.3 \mathrm{mg} / \mathrm{kg}$, respectively) (Fig. $4 \mathrm{~A}-\mathrm{C}$, E). On the other hand, high-dose rapamycin treatment $(1.0 \mathrm{mg} /$ $\mathrm{kg}$ ) significantly decreased the number of p-p38 MAPK-ir cells 

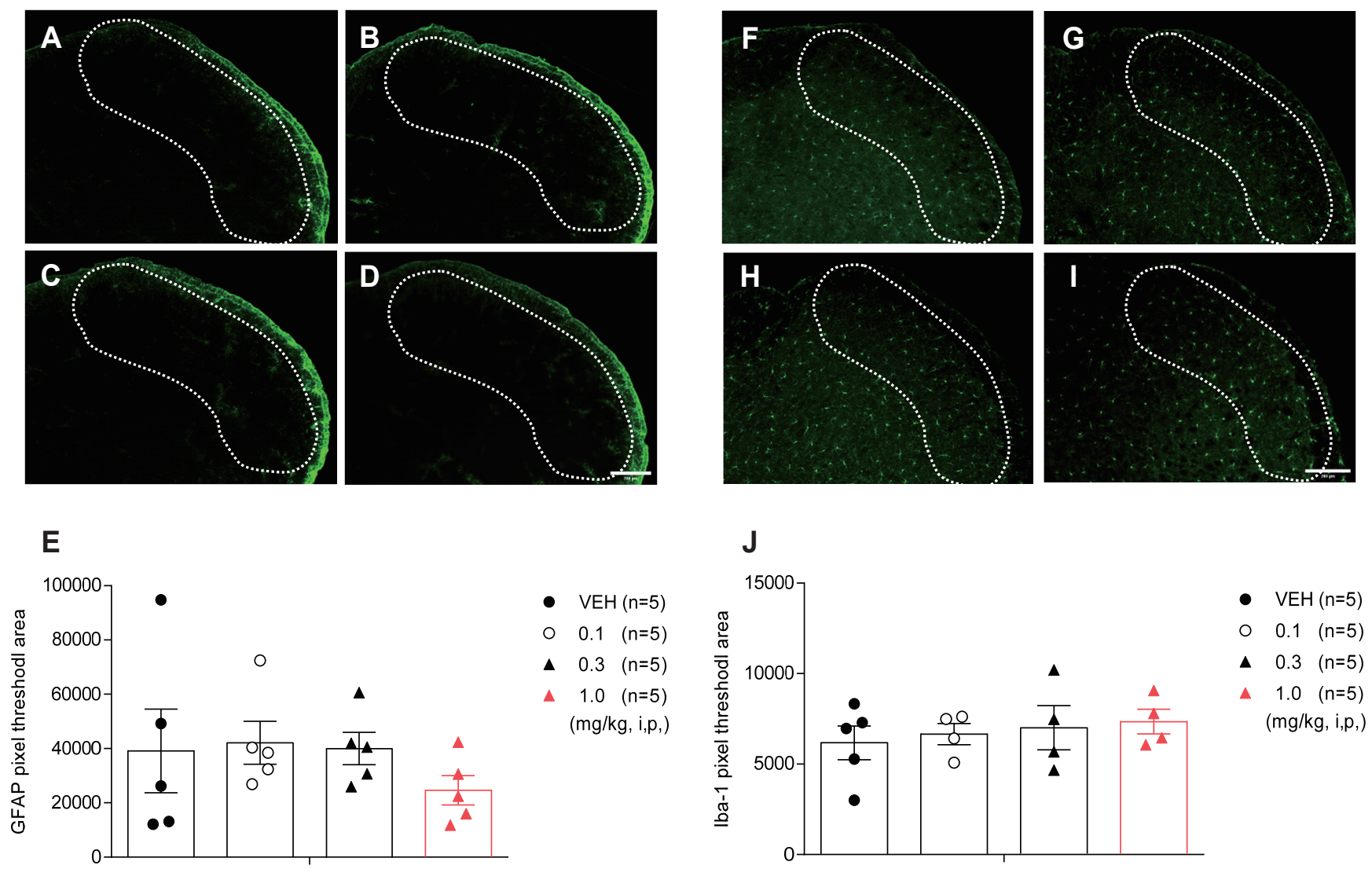

Fig. 3. Effect of rapamycin on glial fibrillary acidic protein (GFAP) and ionized calcium-binding adapter molecule-1 (Iba-1) expression in the trigeminal nucleus caudalis (TNC). The pixel threshold area of either GFAP (A-E) or Iba-1 (F-J) in the TNC remained unchanged in the vehicletreated (VEH) group (A, F) and after all doses of rapamycin treatment $(0.1,0.3$, and $1.0 \mathrm{mg} / \mathrm{kg})(\mathrm{B}-\mathrm{D}, \mathrm{G}-\mathrm{l})$. The boundaries of the TNC are outlined by the dotted line. Scale bar $=200 \mu \mathrm{m}$.

compared with the VEH group (Fig. 4D, E). The arrows in Fig. 4A show the representative p-p38 MAPK-ir cells.

\section{p-p38 MAPK expression is co-localized in microglia, but not neurons or astrocytes in the TNC}

We also investigated the cellular distribution of p-p38 MAPK expression in the TNC and whether it is affected by high-dose rapamycin. As shown in Fig. 5, p-p38 MAPK expression was not shown together with GFAP (Fig. 5A-C) or NeuN (Fig. 5G-I), whereas it was accurately co-localized in Iba-1-ir cells (Fig. 5DF; arrows indicate representative co-localized cells). These results show that high-dose rapamycin treatment $(1.0 \mathrm{mg} / \mathrm{kg})$ reduced p-p38 MAPK expression in TNC microglia, causing an antinociceptive effect in the mouse orofacial formalin test.

\section{DISCUSSION}

The present study demonstrated that i.p. rapamycin treatment had significant dose-dependent antinociceptive effects during the first and second phases of the mice orofacial formalin test. In addition, we showed that the number of Fos-ir cells in the ipsilateral TNC was also reduced in rapamycin-treated mice compared with those in the VEH group. Rapamycin is a valuable drug with various clinical applications, such as the inhibition of mTOR, a protein kinase that controls cell growth by regulating many cellular processes, including protein synthesis and autophagy $[7,26]$. The mTOR is divided into two functionally distinct complexes: mTOR complex 1 (mTORC1) and mTOR complex 2 (mTORC2). Most notably, mTORC1 regulates the activity of peripheral sensory neurons by controlling protein translation; protein synthesis within these sensory neurons also contributes to the nociceptive function in physiological conditions and chronic pain [27]. Rapamycin has recently been reported to produce antinociceptive effects in acute pain models. Asante et al. [28] reported that rapamycin decreased mechanical hypersensitivity in formalin-induced inflammatory rat model. Geranton et al. [29] reported the effect of intrathecal (i.t.) injection of rapamycin in both the acute capsaicin model and spared-nerve injured chronic pain model. In the capsaicin test, i.t. rapamycin significantly reduced mechanical hyperalgesia and allodynia, but not thermal hyperalgesia from 5 $\mathrm{min}$ to $2 \mathrm{~h}$ after capsaicin injection. These studies, when reviewed alongside our results, show that rapamycin injection evokes anti- 

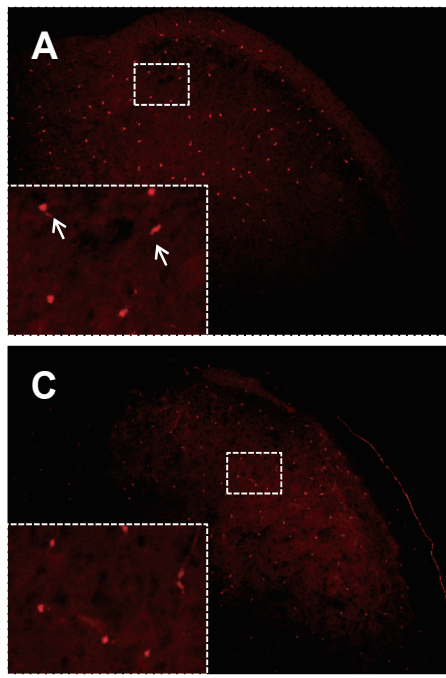
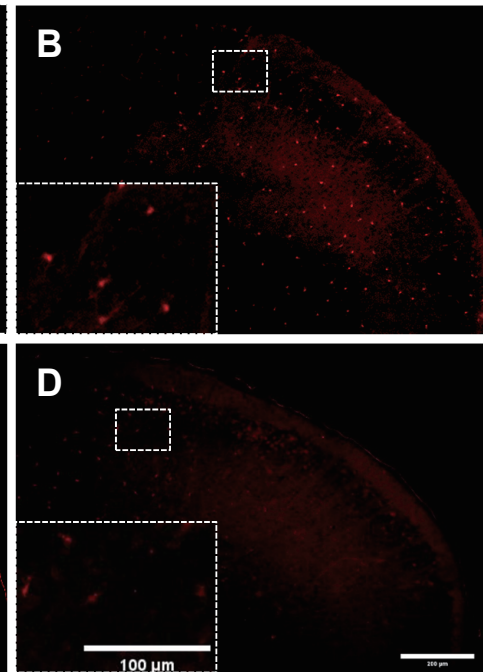

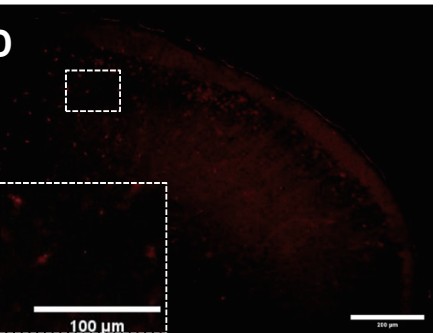

\section{E}

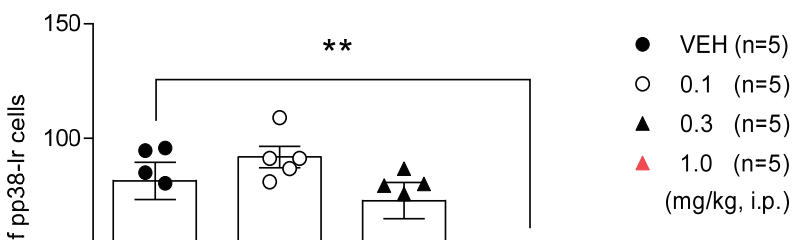

Fig. 4. Effect of rapamycin on phosphorylated p38 (p-p38) mitogen-activated protein kinase (MAPK) in the trigeminal nucleus caudalis (TNC). The expression of p-p38 in the TNC remained unchanged after treatment with low- and medium-dose rapamycin ( 0.1 and $0.3 \mathrm{mg} / \mathrm{kg}$, respectively) (A$C, E ; n=5)$. On the other hand, the number of $p$-p38-ir cells significantly decreased after treatment with high-dose rapamycin $(1.0 \mathrm{mg} / \mathrm{kg})$ compared with the vehicle-treated (VEH) group $\left(D, E_{;}^{* *} p<0.01\right.$, compared with the VEH group, $\left.n=5\right)$. The boundaries of the TNC are outlined by the dotted line. The arrows indicate representative p-p38-ir cells. Scale bar $=200$ and $100 \mu \mathrm{m}$ (magnified panel).

nociceptive effects at similar time periods in two acute tonic pain models (capsaicin test and orofacial formalin test) despite differences in animal species and delivery pathways. It can be said that rapamycin can reduce acute and inflammatory nociceptive responses in the orofacial formalin test. This study is the first to show that mTOR inhibition with rapamycin can relieve orofacial pain in mice.

The change of Fos protein expression in the TNC has a strong positive correlation with the intensity of nociceptive stimulation [30]. Since the trigeminal nervous system is generally activated by peripheral tissue conditions, such as electrical or chemical stimulation of the orofacial or meningeal region (e.g., capsaicin), Fos protein expression has been used as an indicator of the functional activity of neurons in the TNC [31,32]. In this study, we also demonstrated that the orofacial formalin-induced increase of Fos-ir cells in the TNC dose-dependently decreased in the mice treated with rapamycin, implying the close association of the antinociceptive effect of high-dose rapamycin with the modification of orofacial pain processing in the TNC.

Glial cells interact and influence each other's involvement in an exaggerated nociceptive response to injury or inflammation. The activation of astrocytes plays a role in the initiation of acute pain and the maintenance of chronic pain, whereas activated microglia promote pro-inflammatory cytokines or reactive oxygen species, such as TNF- $\alpha$, IL-1 $\beta$, and nitric oxide $[33,34]$. These glia-derived substances cause glia-neuron cross talk, which is associated with the induction of central sensitization, one of the mechanisms of chronic pain [35,36]. Astrocytes were previously found to be activated within $15 \mathrm{~min}$ after s.c. formalin injection into the hindpaw [37]. Astrocyte activation, as identified by increased GFAP expression, was also found in the medullary dorsal horn as early as $1 \mathrm{~h}$ after acute tooth pulp inflammation and within one day after inducing trigeminal chronic inflammation and nerve injury $[38,39]$. On the other hand, significant microglia activation in the spinal cord occurs $24 \mathrm{~h}$ following the injection of zymosan [40], whereas the expression of Ox- 42 , another microglia marker, increased from day 3 to week 4 after s.c. formalin injection into the rat's hindpaw [41]. These studies showed that the activation of astrocytes and microglia has different mechanism of regulation by various extracellular or intracellular signals in the spinal cord or TNC region.

Rapamycin has been reported to suppress the activation of microglia and the increase of pro-inflammatory factors in the CNS [42], and it could also reduce the development of neuropathic pain after spinal cord injury [17]. Because of this, we investigated the changes in the expression of astrocytes and microglia in the TNC after rapamycin treatment in the orofacial formalin test. Interestingly, all doses of rapamycin $(0.1-1.0 \mathrm{mg} / \mathrm{kg})$ used in this study did not alter the expression of GFAP or Iba-1 in the TNC after orofacial formalin injection. This discrepancy between our results and previous studies seems to be due to differences among animal models, specifically regarding pain duration and type (e.g., acute versus chronic pain and inflammatory versus neuropathic pain). Moreover, in contrast to our study, most previous studies involving the activation of microglia or astrocytes in the spinal cord have been performed in chronic pain models [43-45].

Many studies have provided evidence that the MAPK pathway contributes to pain sensitization after tissue and nerve injury via distinct molecular and cellular mechanisms. The phosphorylation of MAPK under different persistent pain conditions results in the induction and maintenance of pain hypersensitivity through transcriptional and non-transcriptional regulation [46]. 


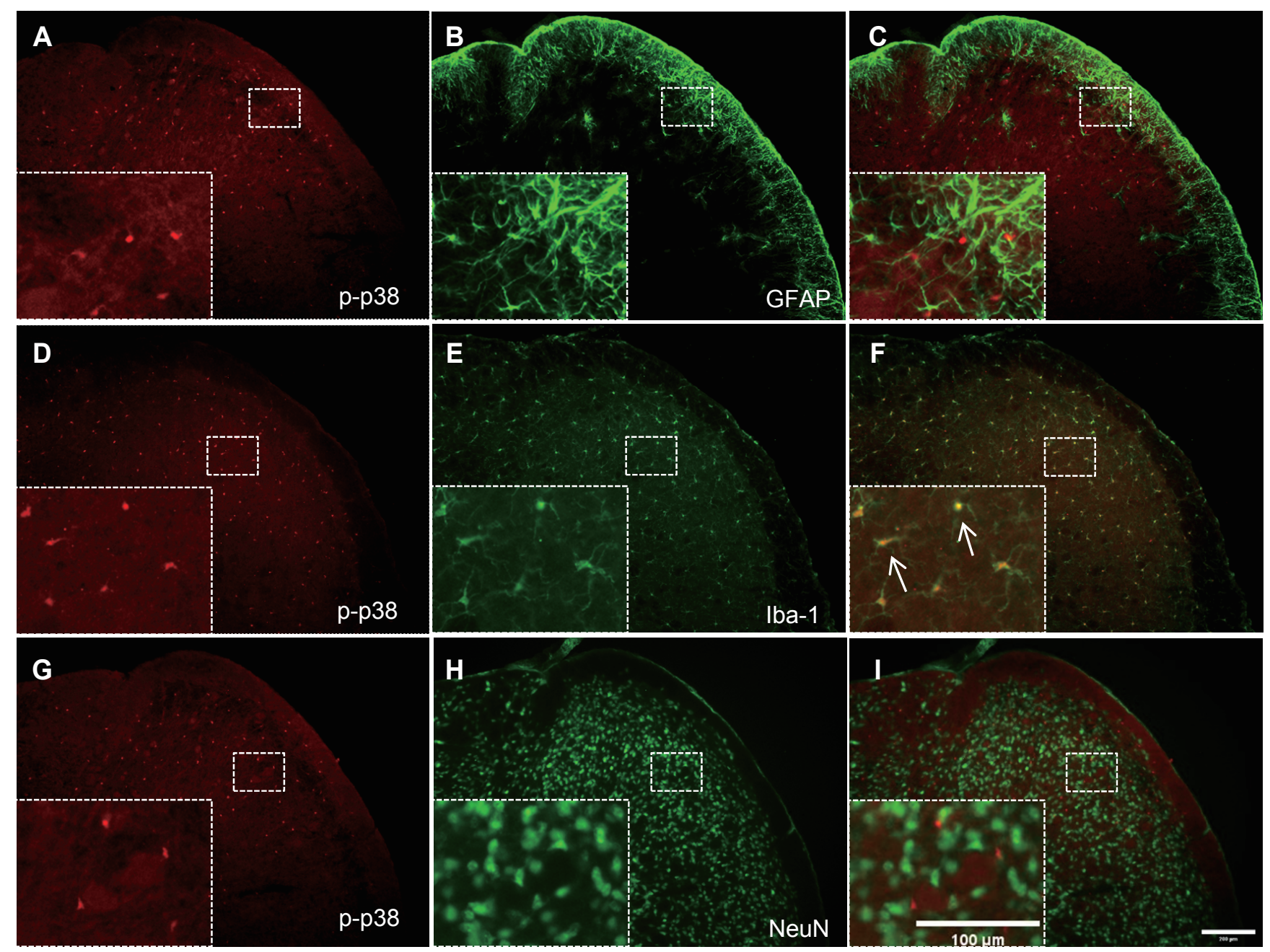

Fig. 5. Co-localization of astrocyte (glial fibrillary acidic protein, GFAP), neuron (neuronal nuclear protein, NeuN), or microglia (ionized calcium-binding adapter molecule-1, lba-1) with phosphorylated p38 (p-p38) MAPK in the trigeminal nucleus caudalis (TNC). Neither GFAP (A-C) nor NeuN (G-I) co-localized with p-p38 in the TNC. In contrast, Iba-1 immunoreactivity was co-expressed in p-p38-positive cells in the TNC in orofacial formalin-injected mice (D-F). The rectangular areas are magnified in panels (C, F, I), whereas arrows indicate representative p-p38-ir cells doublestained with lba-1. Scale bar $=200$ and $100 \mu \mathrm{m}$ (magnified panel).

The MAPK family includes three major members: extracellular signal-regulated kinase, p38, and c-Jun N-terminal kinase, representing three different signaling cascades. In particular, p38 MAPK, which is activated by the upstream kinase MKK3/ MMK6, is considered a stress-inducing kinase, and its phosphorylated form plays an important role in maintaining inflammatory responses and pain hypersensitivities in chronic neuropathic pain models [47,48]. Recently, several studies have indicated that p38 MAPK activation in the spinal microglia mediates pain processing in various acute and chronic pain models, including incision-induced mechanical allodynia [49], peripheral nerve injury [47,50,51], inflammation-evoked pain [52-54] and spinal cord injury [55]. Tateda et al. [17] reported that p38 MAPK activity in the lumbar spine was significantly attenuated by rapamycin treatment and that p-p38 MAPK-positive microglia was relatively reduced. Similarly, we also found that the increased number of p38 MAPK-ir cells in the TNC after orofacial formalin injection was reduced after administering high-dose rapamycin $(1.0 \mathrm{mg} /$ $\mathrm{kg}$ ). Moreover, our immunohistochemical studies showed that p-p38 MAPK-ir cells in the TNC co-localized only with Iba-1, but not with NeuN or GFAP, indicating that the antinociceptive effect of high-dose rapamycin might be caused by the regulation of microglial p-p38 MAPK in the TNC.

Understanding the differential role of Fos and p-p38 protein in this study is also important. As mentioned earlier, the Fos protein is commonly used as a neuronal activation marker for nociception; therefore, this reduction in Fos expression may be a secondary change in the analgesic effect of rapamycin. On the other hand, for p-p38, it has been reported that the activation of the Srcl p38 MAPK signaling cascade in spinal microglial cells contributed to late-stage persistent mechanical hyperalgesia induced by formalin injection into the paw [56]. Several reports have shown that the increased expression of p-p38 was closely associated with different types of pain induction $[14,15,57]$ and that the pharma- 
cological inhibition of p38 MAPK reduces mechanical allodynia in neuropathic pain models $[58,59]$. Therefore, in this experiment, we suspect that $\mathrm{p} 38 \mathrm{MAPK}$ activation is involved in pain induction and that rapamycin treatment inhibits p38 MAPK activation, thus exerting antinociceptive effects in the inflammatory acute pain model. However, how rapamycin treatment modulates the activity (i.e., phosphorylation) of p38 MAPK in the microglia of the TNC and whether this is associated with the suppression of orofacial chronic pain conditions, including trigeminal neuralgia and temporomandibular joint disorders, remain unclear. Further studies using animal models of trigeminal neuropathic pain or chronic mandibular inflammation are needed to address those concerns.

In conclusion, the present study demonstrated that rapamycin dose-dependently reduced nociceptive responses in both the first and second phases of the orofacial formalin test. The number of For-ir cells in the ipsilateral TNC was also reduced by rapamycin treatment compared with those in the VEH group. Moreover, the number of p-p38-ir cells in the ipsilateral TNC significantly decreased after treatment with high-dose rapamycin, and this p-p38 expression was co-localized in the microglia, but not in neurons and astrocytes. In summary, these findings suggest that rapamycin, an mTOR inhibitor, reduces orofacial pain and that microglial p-p38 MAPK inhibition can be associated with its antinociceptive action in the orofacial region.

\section{ACKNOWLEDGEMENTS}

This study was supported by the National Research Foundation of Korea (NRF) grants funded by the Korea government (MSIP) (no. NRF-2019R1F1A1063430). This work was also supported by a grant from Kyung Hee University in 2018 (KHU-20181069).

\section{CONFLICTS OF INTEREST}

The authors declare no conflicts of interest.

\section{REFERENCES}

1. Raboisson P, Dallel R. The orofacial formalin test. Neurosci Biobehav Rev. 2004;28:219-226.

2. Miranda HF, Sierralta F, Prieto JC. Synergism between NSAIDs in the orofacial formalin test in mice. Pharmacol Biochem Behav. 2009;92:314-318.

3. Muñoz J, Navarro C, Noriega V, Pinardi G, Sierralta F, Prieto JC, Miranda HF. Synergism between COX-3 inhibitors in two animal models of pain. Inflammopharmacology. 2010;18:65-71.

4. Murakami M, Ichisaka T, Maeda M, Oshiro N, Hara K, Edenhofer F, Kiyama H, Yonezawa K, Yamanaka S. mTOR is essential for growth and proliferation in early mouse embryos and embryonic stem cells.
Mol Cell Biol. 2004;24:6710-6718.

5. Zoncu R, Efeyan A, Sabatini DM. mTOR: from growth signal integration to cancer, diabetes and ageing. Nat Rev Mol Cell Biol. 2011;12:21-35.

6. Altmann C, Hardt S, Fischer C, Heidler J, Lim HY, Häussler A, Albuquerque B, Zimmer B, Möser C, Behrends C, Koentgen F, Wittig I, Schmidt MHH, Clement AM, Deller T, Tegeder I. Progranulin overexpression in sensory neurons attenuates neuropathic pain in mice: role of autophagy. Neurobiol Dis. 2016;96:294-311.

7. Guertin DA, Sabatini DM. An expanding role for mTOR in cancer. Trends Mol Med. 2005;11:353-361.

8. Feng T, Yin Q, Weng ZL, Zhang JC, Wang KF, Yuan SY, Cheng W. Rapamycin ameliorates neuropathic pain by activating autophagy and inhibiting interleukin-1 $\beta$ in the rat spinal cord. J Huazhong Univ Sci Technolog Med Sci. 2014;34:830-837.

9. Obara I, Medrano MC, Signoret-Genest J, Jiménez-Díaz L, Géranton SM, Hunt SP. Inhibition of the mammalian target of rapamycin complex 1 signaling pathway reduces itch behaviour in mice. Pain. 2015;156:1519-1529.

10. Xu Q, Fitzsimmons B, Steinauer J, O'Neill A, Newton AC, Hua XY, Yaksh TL. Spinal phosphinositide 3-kinase-Akt-mammalian target of rapamycin signaling cascades in inflammation-induced hyperalgesia. J Neurosci. 2011;31:2113-2124.

11. Ji RR, Berta T, Nedergaard M. Glia and pain: is chronic pain a gliopathy? Pain. 2013;154 Suppl 1:S10-S28.

12. Kreutzberg GW. Microglia: a sensor for pathological events in the CNS. Trends Neurosci. 1996;19:312-318.

13. Shinoda M, Hayashi Y, Kubo A, Iwata K. Pathophysiological mechanisms of persistent orofacial pain. J Oral Sci. 2020;62:131-135.

14. Ji RR, Woolf CJ. Neuronal plasticity and signal transduction in nociceptive neurons: implications for the initiation and maintenance of pathological pain. Neurobiol Dis. 2001;8:1-10.

15. Ji RR, Samad TA, Jin SX, Schmoll R, Woolf CJ. p38 MAPK activation by NGF in primary sensory neurons after inflammation increases TRPV1 levels and maintains heat hyperalgesia. Neuron. 2002;36:57-68.

16. Sekiguchi A, Kanno H, Ozawa H, Yamaya S, Itoi E. Rapamycin promotes autophagy and reduces neural tissue damage and locomotor impairment after spinal cord injury in mice. J Neurotrauma. 2012; 29:946-956.

17. Tateda S, Kanno H, Ozawa H, Sekiguchi A, Yahata K, Yamaya S, Itoi E. Rapamycin suppresses microglial activation and reduces the development of neuropathic pain after spinal cord injury. J Orthop Res. 2017;35:93-103.

18. Bornhof M, Ihmsen H, Schwilden H, Yeomans DC, Tzabazis A. The orofacial formalin test in mice revisited--effects of formalin concentration, age, morphine and analysis method. J Pain. 2011;12:633-639.

19. Roh DH, Yoon SY. Sigma-1 receptor antagonist, BD1047 reduces nociceptive responses and phosphorylation of $\mathrm{p} 38$ MAPK in mice orofacial formalin model. Biol Pharm Bull. 2014;37:145-151.

20. Roh DH, Kim HW, Yoon SY, Kang SY, Kwon YB, Cho KH, Han HJ, Ryu YH, Choi SM, Lee HJ, Beitz AJ, Lee JH. Bee venom injection significantly reduces nociceptive behavior in the mouse formalin test via capsaicin-insensitive afferents. J Pain. 2006;7:500-512.

21. Müller R, Bravo R, Burckhardt J, Curran T. Induction of c-fos gene and protein by growth factors precedes activation of c-myc. Nature. 1984;312:716-720. 
22. Williams S, Evan GI, Hunt SP. Changing patterns of c-fos induction in spinal neurons following thermal cutaneous stimulation in the rat. Neuroscience. 1990;36:73-81.

23. Doyle T, Chen Z, Muscoli C, Obeid LM, Salvemini D. Intraplantarinjected ceramide in rats induces hyperalgesia through an NF$\kappa \mathrm{B}$ - and p38 kinase-dependent cyclooxygenase 2/prostaglandin E2 pathway. FASEB J. 2011;25:2782-2791.

24. Wang H, Tian J, Du F, Wang T. Effect of peritoneal transport characteristics on clinical outcome in nondiabetic and diabetic nephropathy patients with peritoneal dialysis. Iran J Kidney Dis. 2019;13:5666.

25. Choi SR, Beitz AJ, Lee JH. Inhibition of cytochrome P450c17 reduces spinal astrocyte activation in a mouse model of neuropathic pain via regulation of p38 MAPK phosphorylation. Biomed Pharmacother. 2019;118:109299.

26. Shillingford JM, Murcia NS, Larson CH, Low SH, Hedgepeth R, Brown N, Flask CA, Novick AC, Goldfarb DA, Kramer-Zucker A, Walz G, Piontek KB, Germino GG, Weimbs T. The mTOR pathway is regulated by polycystin-1, and its inhibition reverses renal cystogenesis in polycystic kidney disease. Proc Natl Acad Sci U S A. 2006; 103:5466-5471.

27. Obara I, Hunt SP. Axonal protein synthesis and the regulation of primary afferent function. Dev Neurobiol. 2014;74:269-278.

28. Asante CO, Wallace VC, Dickenson AH. Formalin-induced behavioural hypersensitivity and neuronal hyperexcitability are mediated by rapid protein synthesis at the spinal level. Mol Pain. 2009;5:27.

29. Géranton SM, Jiménez-Díaz L, Torsney C, Tochiki KK, Stuart SA, Leith JL, Lumb BM, Hunt SP. A rapamycin-sensitive signaling pathway is essential for the full expression of persistent pain states. $J$ Neurosci. 2009;29:15017-15027.

30. Ter Horst GJ, Meijler WJ, Korf J, Kemper RH. Trigeminal nociception-induced cerebral Fos expression in the conscious rat. Cephalalgia. 2001;21:963-975.

31. Mitsikostas DD, Sanchez del Rio M. Receptor systems mediating cfos expression within trigeminal nucleus caudalis in animal models of migraine. Brain Res Brain Res Rev. 2001;35:20-35.

32. Bhatt DK, Gupta S, Ploug KB, Jansen-Olesen I, Olesen J. mRNA distribution of CGRP and its receptor components in the trigeminovascular system and other pain related structures in rat brain, and effect of intracerebroventricular administration of CGRP on Fos expression in the TNC. Neurosci Lett. 2014;559:99-104.

33. Beggs S, Salter MW. Stereological and somatotopic analysis of the spinal microglial response to peripheral nerve injury. Brain Behav Immun. 2007;21:624-633.

34. Romero-Sandoval A, Chai N, Nutile-McMenemy N, Deleo JA. A comparison of spinal Ibal and GFAP expression in rodent models of acute and chronic pain. Brain Res. 2008;1219:116-126.

35. Tsuda M, Inoue K, Salter MW. Neuropathic pain and spinal microglia: a big problem from molecules in "small" glia. Trends Neurosci. 2005;28:101-107.

36. Chang YW, Tan A, Saab C, Waxman S. Unilateral focal burn injury is followed by long-lasting bilateral allodynia and neuronal hyperexcitability in spinal cord dorsal horn. J Pain. 2010;11:119-130.

37. Guo W, Wang H, Watanabe M, Shimizu K, Zou S, LaGraize SC, Wei F, Dubner R, Ren K. Glial-cytokine-neuronal interactions underlying the mechanisms of persistent pain. J Neurosci. 2007;27:60066018.
38. Piao ZG, Cho IH, Park CK, Hong JP, Choi SY, Lee SJ, Lee S, Park $\mathrm{K}$, Kim JS, Oh SB. Activation of glia and microglial p38 MAPK in medullary dorsal horn contributes to tactile hypersensitivity following trigeminal sensory nerve injury. Pain. 2006;121:219-231.

39. Okada-Ogawa A, Suzuki I, Sessle BJ, Chiang CY, Salter MW, Dostrovsky JO, Tsuboi Y, Kondo M, Kitagawa J, Kobayashi A, Noma N, Imamura Y, Iwata K. Astroglia in medullary dorsal horn (trigeminal spinal subnucleus caudalis) are involved in trigeminal neuropathic pain mechanisms. J Neurosci. 2009;29:11161-11171.

40. Clark AK, Gentry C, Bradbury EJ, McMahon SB, Malcangio M. Role of spinal microglia in rat models of peripheral nerve injury and inflammation. Eur J Pain. 2007;11:223-230.

41. Fu KY, Light AR, Matsushima GK, Maixner W. Microglial reactions after subcutaneous formalin injection into the rat hind paw. Brain Res. 1999;825:59-67.

42. Dello Russo C, Lisi L, Tringali G, Navarra P. Involvement of mTOR kinase in cytokine-dependent microglial activation and cell proliferation. Biochem Pharmacol. 2009;78:1242-1251.

43. Inyang KE, Szabo-Pardi T, Wentworth E, McDougal TA, Dussor G, Burton MD, Price TJ. The antidiabetic drug metformin prevents and reverses neuropathic pain and spinal cord microglial activation in male but not female mice. Pharmacol Res. 2019;139:1-16.

44. Tozaki-Saitoh H, Masuda J, Kawada R, Kojima C, Yoneda S, Masuda T, Inoue K, Tsuda M. Transcription factor MafB contributes to the activation of spinal microglia underlying neuropathic pain development. Glia. 2019;67:729-740.

45. Lopes F, Vicentini FA, Cluny NL, Mathews AJ, Lee BH, Almishri WA, Griffin L, Gonçalves W, Pinho V, McKay DM, Hirota SA, Swain MG, Pittman QJ, Sharkey KA. Brain TNF drives post-inflammation depression-like behavior and persistent pain in experimental arthritis. Brain Behav Immun. 2020;89:224-232.

46. Ma W, Quirion R. Partial sciatic nerve ligation induces increase in the phosphorylation of extracellular signal-regulated kinase (ERK) and c-Jun N-terminal kinase (JNK) in astrocytes in the lumbar spinal dorsal horn and the gracile nucleus. Pain. 2002;99:175-184.

47. Jin SX, Zhuang ZY, Woolf CJ, Ji RR. p38 mitogen-activated protein kinase is activated after a spinal nerve ligation in spinal cord microglia and dorsal root ganglion neurons and contributes to the generation of neuropathic pain. J Neurosci. 2003;23:4017-4022.

48. Hains BC, Waxman SG. Activated microglia contribute to the maintenance of chronic pain after spinal cord injury. J Neurosci. 2006;26:4308-4317.

49. Wen YR, Suter MR, Ji RR, Yeh GC, Wu YS, Wang KC, Kohno T, Sun WZ, Wang CC. Activation of p38 mitogen-activated protein kinase in spinal microglia contributes to incision-induced mechanical allodynia. Anesthesiology. 2009;110:155-165.

50. Gu YW, Su DS, Tian J, Wang XR. Attenuating phosphorylation of p38 MAPK in the activated microglia: a new mechanism for intrathecal lidocaine reversing tactile allodynia following chronic constriction injury in rats. Neurosci Lett. 2008;431:129-134.

51. Suter MR, Berta T, Gao YJ, Decosterd I, Ji RR. Large A-fiber activity is required for microglial proliferation and p38 MAPK activation in the spinal cord: different effects of resiniferatoxin and bupivacaine on spinal microglial changes after spared nerve injury. Mol Pain. 2009;5:53.

52. Svensson CI, Marsala M, Westerlund A, Calcutt NA, Campana WM, Freshwater JD, Catalano R, Feng Y, Protter AA, Scott B, Yaksh 
TL. Activation of p38 mitogen-activated protein kinase in spinal microglia is a critical link in inflammation-induced spinal pain processing. J Neurochem. 2003;86:1534-1544.

53. Cui XY, Dai Y, Wang SL, Yamanaka H, Kobayashi K, Obata K, Chen J, Noguchi K. Differential activation of p38 and extracellular signalregulated kinase in spinal cord in a model of bee venom-induced inflammation and hyperalgesia. Mol Pain. 2008;4:17.

54. Nasseri B, Zaringhalam J, Daniali S, Manaheji H, Abbasnejad Z, Nazemian $V$. Thymulin treatment attenuates inflammatory pain by modulating spinal cellular and molecular signaling pathways. Int Immunopharmacol. 2019;70:225-234.

55. Crown ED, Gwak YS, Ye Z, Johnson KM, Hulsebosch CE. Activation of p38 MAP kinase is involved in central neuropathic pain following spinal cord injury. Exp Neurol. 2008;213:257-267.

56. Tan YH, Li K, Chen XY, Cao Y, Light AR, Fu KY. Activation of Src family kinases in spinal microglia contributes to formalin-induced persistent pain state through p38 pathway. J Pain. 2012;13:1008-1015.

57. Kumar S, Boehm J, Lee JC. p38 MAP kinases: key signalling molecules as therapeutic targets for inflammatory diseases. Nat Rev Drug Discov. 2003;2:717-726.

58. Obata K, Yamanaka H, Kobayashi K, Dai Y, Mizushima T, Katsura H, Fukuoka T, Tokunaga A, Noguchi K. Role of mitogen-activated protein kinase activation in injured and intact primary afferent neurons for mechanical and heat hypersensitivity after spinal nerve ligation. J Neurosci. 2004;24:10211-10222.

59. Lim EJ, Jeon HJ, Yang GY, Lee MK, Ju JS, Han SR, Ahn DK. Intracisternal administration of mitogen-activated protein kinase inhibitors reduced mechanical allodynia following chronic constriction injury of infraorbital nerve in rats. Prog Neuropsychopharmacol Biol Psychiatry. 2007;31:1322-1329. 